\title{
Iris ultrastructure in patients with synechiae as revealed by in vivo laser scanning confocal microscopy
}

\section{In vivo iris ultrastructure in patients with Synechiae by Laser Scanning Confocal Microscopy}

\author{
Ming Li $\mathrm{Li}^{1,2,3}$, Hongbo Cheng ${ }^{1}$, Ping Guo ${ }^{1}$, Chun Zhang ${ }^{1}$, Song Tang ${ }^{1}$ and Shusheng Wang ${ }^{2,3^{*}}$
}

\begin{abstract}
Background: Iris plays important roles in ocular physiology and disease pathogenesis. Currently it is technically challenging to noninvasively examine the human iris ultrastructure in vivo. The purpose of the current study is to reveal human iris ultrastructure in patients with synechiae by using noninvasive in vivo laser scanning confocal microscopy (LSCM).

Methods: The ultrastructure of iris in thirty one patients, each with synechiae but transparent cornea, was examined by in vivo LSCM.

Results: Five characteristic iris ultrastructures was revealed in patients with synechiae by in vivo LSCM, which include: 1. tree trunk-like structure; 2. tree branch/bush-like structure; 3. Fruit-like structure; 4. Epithelioid-like structure; 5 . deep structure. Pigment granules can be observed as a loose structure on the top of the arborization structure. In iris-associated diseases with Tyndall's Phenomenon and keratic precipitates, the pigment particles are more likely to fall off from the arborization structure.

Conclusions: The ultrastructure of iris in patients with synechiae has been visualized using in vivo LSCM. Five iris ultrastructures can be clearly observed, with some of the structures maybe disease-associated. The fall-off of the pigment particles may cause the Tyndall's Phenomenon positive. In vivo LSCM provides a non-invasive approach to observe the human iris ultrastructure under certain eye disease conditions, which sets up a foundation to visualize certain iris-associated diseases in the future.
\end{abstract}

Keywords: Iris, In vivo, Ultrastructure, Laser scanning confocal microscopy

\section{Background}

The human iris is a pigmented membranous structure located between the cornea and the lens. It has important physiological functions, including pupil size adjustment and participation in the formation of the eye darkroom structure. It also plays an important role the pathogenesis of many ocular diseases, such as uveitis

\footnotetext{
* Correspondence: swang1@tulane.edu

2Department of Cell and Molecular Biology, Tulane University, 2000 Percival Stern Hall, 6400 Freret Street, New Orleans, LA 70118, USA

${ }^{3}$ Department of Ophthalmology, Tulane University, 1430 Tulane Avenue, SL-69, New Orleans, LA 70112, USA

Full list of author information is available at the end of the article
}

and glaucoma, among others [1-4]. By histology, based on the sections from embedded pathological samples, the iris can be divided into four-layers, including the anterior limiting layer, the iris stroma and the pupil sphincter, anterior pigment myoepithelium and pupil dilatator muscle, as well as the posterior pigment epithelium. In order to test the involvement of iris in ocular disease, it would be ideal to develop a noninvasive method to examine the human iris ultrastructure.

In vivo laser scanning confocal microscopy (LSCM) has been used to visualize the layers of corneal structures at the cellular level. It has been applied clinically to

\section{C)


reveal characteristic features in many keratopathies [5-13]. Compared to the conventional imaging methods, in vivo LSCM can achieve superior vertical and lateral resolutions and provide outstanding image contrast, which allow for rapid real-time in vivo examination of corneal ultrastructures. However, under normal conditions, the distance of the iris from the cornea prevents the visualization of iris ultrastructure using in vivo LSCM, even in the peripheral/limbal region [2-5]. Some researchers have tried to optimize in vivo LSCM imaging by changing the camera lens and increasing its working distance, but the quality of images was still subpar [14].

To understand the ultrastructure of the iris in vivo and study its relationship to iris-associated diseases, an approach have been devised to visualize iris ultrastructure using in vivo LSCM in 31 special patients who had anterior synechiae (iris-cornea contact) due to various reasons. Characteristic ultrastructures of human synechiae iris were observed through the transparent corneas of these patients. These data represent high quality noninvasive imaging of synechiae iris ultrastructures. Moreover, some of the features may be associated with the disease conditions.

\section{Methods}

\section{Patients}

The study was conducted in compliance with informed consent regulations and the Declaration of Helsinki. The study protocol was approved by internal review board (IRB) of Shenzhen Eye Hospital. Informed consent was obtained from the patients for the study. Eyes from 31 patients in Shenzhen Eye Hospital (Shenzhen city, P. R. China) who had synechiae but transparent cornea were examined during the period from March 2008 to March 2014. These includes14 women and17 men with an average age of $34.8 \pm 5.6$ years old. The causes of contact between the iris and the cornea in the patients include: eight cases of post-traumatic iridocorneal adhesions, five cases of uveitis, seven cases of iridocorneal endothelial (ICE) syndrome, three cases of angle-closure glaucoma, four cases of corneal transplantation, four cases of posteye surgery with contacting of the iris and the cornea.

All patients were first examined and diagnosed by an ophthalmologist specialized in studying iris and cornea contact. Data collection included medical and ophthalmological history, age, age at diagnosis, gender, detailed slitlamp examination and in vivo LSCM imaging. At the time of in vivo LSCM examination, the eye conditions were stable. All individual patients were informed of the aims of recording these data, including in vivo LSCM imaging.

\section{In vivo laser scanning confocal microscopy}

The area with contacting of the iris and the cornea was examined by in vivo LSCM with magnification up to
X800 (HRT II Rostock Cornea Module, diode laser $670 \mathrm{~nm}$, Heidelberg Engineering $\mathrm{GmbH}$, Germany). Images consist of $384 \mathrm{X} 384$ pixels covering an area of $400 \mathrm{X} 400 \mathrm{~mm}$ with a transverse optical resolution of approximately $1 \mathrm{~mm} /$ pixel and an acquisition time of 0.024 s (Heidelberg Engineering).

Before microscopy examination, a drop of topical anesthetic (0.5 \% Alcaine, Alcon Corp, Fort Worth, Texas USA) was applied to the lower conjunctival sac of the patients. A sterile Tomocap (Heidelberg Engineering) was mounted over the objective of the microscope (Zeiss, Jena, Germany; 363), and a drop of Vidisic gel (0.2\% Carbomer 940; Bausch \& Lomb, Germany) was used as a coupling agent between the cap and the lens objective.

The patient's head was positioned in the headstock with the eyes steadily gazed at the fixation tool. The area with contacting of the iris and the cornea was examined and images were recorded at one point along the $\mathrm{z}$-axis as single scans or in the movie motion mode. Each eye was examined for less than $5 \mathrm{~min}$. To standardize measurements, all images were subsequently randomized and encoded by a single independent observer.

\section{Results}

By examining patients with iris-cornea contact, characteristic ultrastructures of human synechiae iris were detected under in vivo LSCM. The ultrastructure of human synechiae iris in vivo under the laser scanning confocal microscope resembles a tree-like structure that branches from the surface of the iris that contacts the cornea to the deeper interior. The structure gradually branches from tree trunk-like structure to tree branch or bush-like structure, then to fruit-like structure, and finally to epithelioid-like structure and deep structure. A lot of pigment clumps were observed to attach to different structures. Details of the structures are described below.

1. Tree trunk-like structure (Fig. 1a-c). This structure was observed on the surface of iris that contacts the cornea. It is a support structure with very thick arrangement, similar to a tree trunk. It showed moderate to high light reflection with no cellular structure observed under in vivo LSCM. Scattered highly light-reflective pigment clumps could be observed near the tree trunks.

2. Tree branch/bush-like structure (Fig. 2a-c). Tree branch-like structure was observed under the surface of the iris by in vivo LSCM. It has a tree branch-like or fine arborization shape. Pigment agglomerates could either be clearly seen (Fig. 2a) or not seen (Fig. 2b). Tree bush-like structure was observed from the branching of the tree branch-like structure (Fig. 2c). This structure can be associated with or without fruit-like pigment agglomerates. 

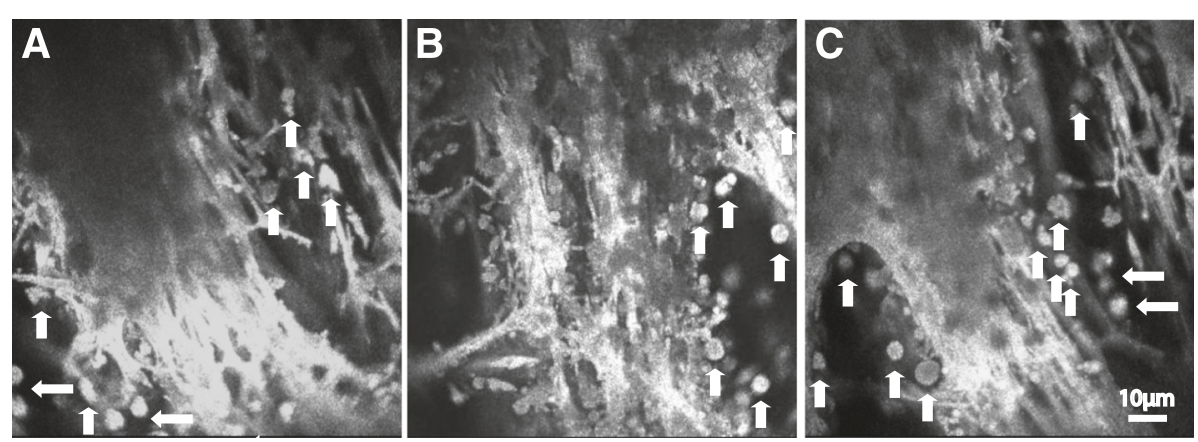

Fig. 1 Tree trunk-like structure structures (a-c) in the human iris with synechiae by in vivo LSCM. Note the support structure with very thick arrangement, similar to the trunk with moderate to high reflection. On the left side and below, finer tree branch-like structure is visible with few scattered pigment clumps. Scale bar equals to $10 \mu \mathrm{m}$. Arrows show the pigment granules

3. Fruit-like structure (Fig. 3a-b). This structure has the fruit-like pigment agglomerates and was observed at the top of the tree bush-like structure.

4. Epithelioid-like structure (Fig. 4a-b). In this structure, the epithelioid-like cells are arranged in sheets. The cell borders were highly light-reflective, while the structures inside the cells were low to medium light-reflective under in vivo LSCM. Little pigment granules could be observed at the top of epithelioid-like cells.

5. Deep structures (Fig. 5a-b). These structures were observed at the deep interior of iris by in vivo LSCM. They could be either coarse or lobular. Due to the limited penetration of the laser, so the structures were more blurred.

In sum, large number of dendritic scaffold structures can be observed on the iris surface by in vivo LSCM. They grow and extend like tree branches, tapering gradually from coarse to fine branches. With branching, the corresponding pigment clumps and particles are increasing gradually, forming clusters of fruit structure similar to hanging bracket tip. For example, there are few pigment granules located at the tree-trunk and branch-like structures in Fig. 1a-b. However, many pigment granules were seen in the epitheliod-like structure in Fig. 4. In a variety of eye diseases with pigment particles falling from iris known as Tyndall's Phenomenon and keratic precipitates, the pigment particles are likely to fall off from the branches. This can be observed in Figs. 1, 4 and 5 (showing pigment particles falling off).

Statistically, the percentage of different structures were found to be different in different patients (Fig. 6 and Additional file 1: Table S1). While tree trunk-like structure, tree branch/bush-like structure and fruit like structure were found in more than $90 \%$ of the patients with synechiae, the epitheloid-like and deep structures were observed in about $70 \%$ of the patients. In 3 of 8 cases of post-traumatic iridocorneal adhesions and 2 of 7 cases of iridocorneal endothelial syndrome, the epitheliod-like and deep structures were not observed. Therefore, the 5 types of structures were not necessarily observed in every patient, which may due to the technical limitation of the in vivo LSCM under the disease conditions.
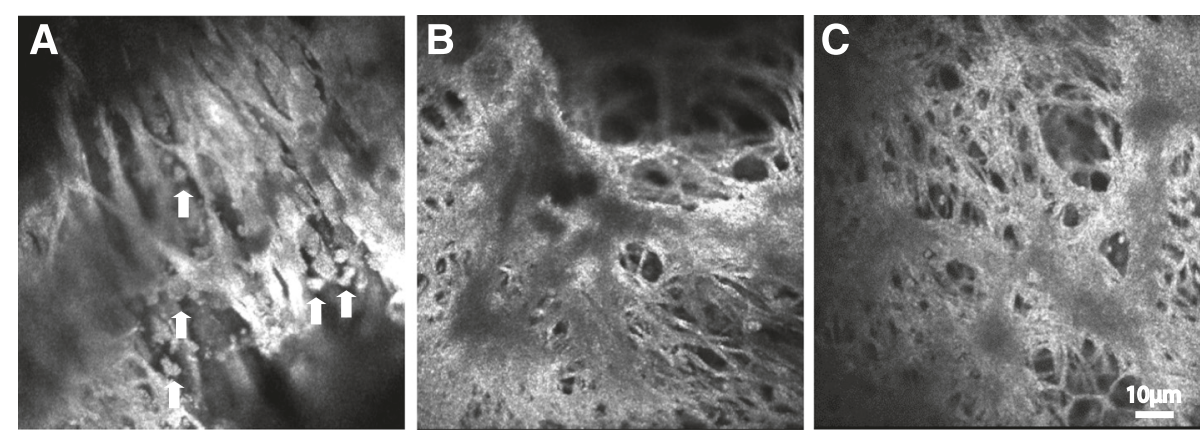

Fig. 2 Tree branch-like structures. a Tree branch-like structure with fine dendritic structure with clear pigment agglomerates. b Tree branch-like structure without pigment agglomerates; c Tree bush-like structure branching from the tree branch-like structure. Scale bar equals to $10 \mu \mathrm{m}$. Arrows show the pigment granules 

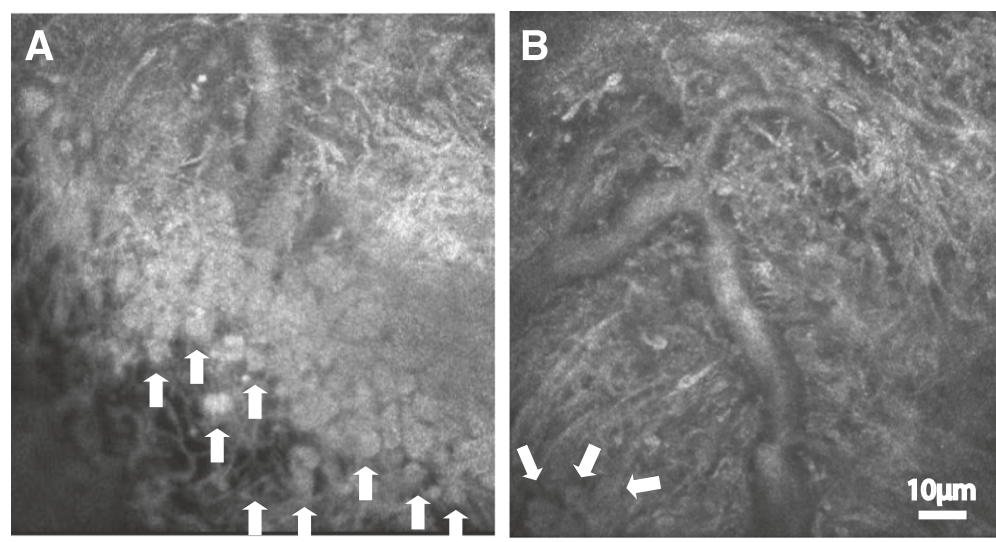

Fig. 3 a-b Fruit-like structures showing pigment agglomerates in the tree bush-like structure. Scale bar equals to $10 \mu m$. Arrows show the pigment granules

\section{Discussion}

The iris is a disc-shaped membrane structure in the front-most portion of the uveal. The center of the disc is called pupil, which can adjust the amount of light entering the eye. Iris plays an important role in many eye diseases $[15,16]$. Clinically, to observe iris, the most common method is to use slit lamp microscope. New technologies, such as UBM (ultrasound biomicroscopy), OCT (optical coherence tomography), are also used in clinical observation of the iris nowadays [17]. However, the resolution of these technologies is limited, and is not suitable for observing iris ultrastructures.

In vivo LSCM can magnify images for up to 800 times, representing an excellent tool to visualize iris ultrastructure. Due to the working distance, the iris structure cannot be observed by in vivo LSCM under normal circumstances since the iris is away from the cornea $[18,19]$. Even when the Nikon lens of in vivo LSCM was changed to increase its working distance, the acquired iris image was still not clear [14].
We took advantage of the patients with iris-cornea contact which were caused by trauma, eye surgery and diseases. By adjusting the focal length of in vivo LSCM, as well as the laser through the cornea into the iris contacting, we successfully obtained high resolution human synechiae iris ultrastructure [20-22].

By in vivo LSCM, The ultrastructure of iris can be divided into the following five characteristic types: (1) tree trunk-like structure (Fig. 1a); (2) tree branch/bush-like structure (Fig. 1b-d); (3) Fruit-like structure (Fig. 1e); (4) epithelioid-like structure (Fig. 1f); and (5) deep structure (Fig. 1g-h). These structures are different from and cannot substitute what described from the histopathological structures of the iris after conventional sample dehydration, embedding and sectioning, but they represent the real-time and live-cell iris structure in a non-invasive condition. Of note, it is currently difficult to directly correlate the five characteristic structures in our noninvasive in vivo studies with the sections from the histopathological studies.
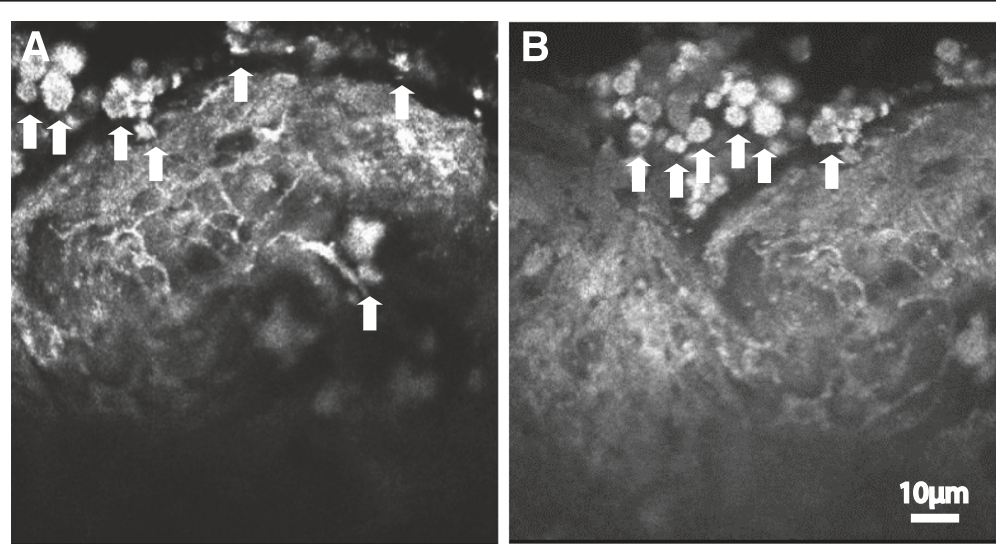

Fig. 4 a-b Epithelioid-like structure that epithelioid cells arranged in sheets. Pigment granules can be seen at the right top of epithelioid cells. Scale bar equals to $10 \mu \mathrm{m}$. Arrows show the pigment granules 

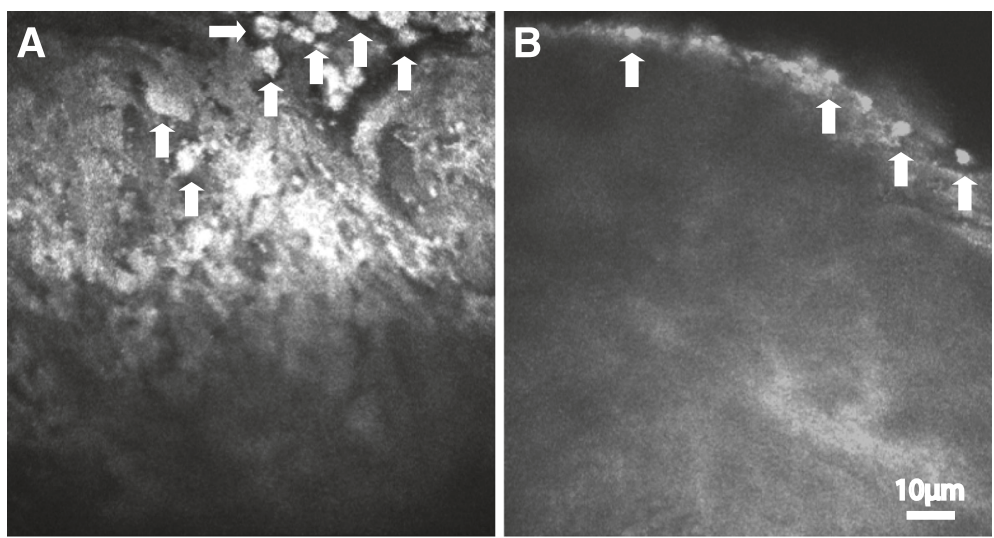

Fig. 5 a-b Deep structure that is coarse or lobulating. Scale bar equals to $10 \mu \mathrm{m}$. Arrows show the pigment granules

We found that the support structure of the iris branches gradually from coarse to fine. Based on the size of the support structure, the stent surface of iris can be divided into three classes of structures: tree trunk-, tree branch/bush- and fruit-like structure. Epitheliod-like structure, which we define as the class 4 structure, is formed on the epithelial surface at the base of the stentlike structure. Even with our special samples, due to the existence of numerous iris pigment particles and the support structure, as well as the limited penetration of the laser, the ultrastructure of the iris deep imaging is still not clear, which represents a limitation of this technology [8]. The shallower in position of the iris, the clearer is the image. On the contrary, the deeper in position, the blurrier the image. We define these deep structures as class 5 structure. We also observed a lot of pigments in the iris covering the stent surface, and the pigment particles stand up at the tip. We found in some

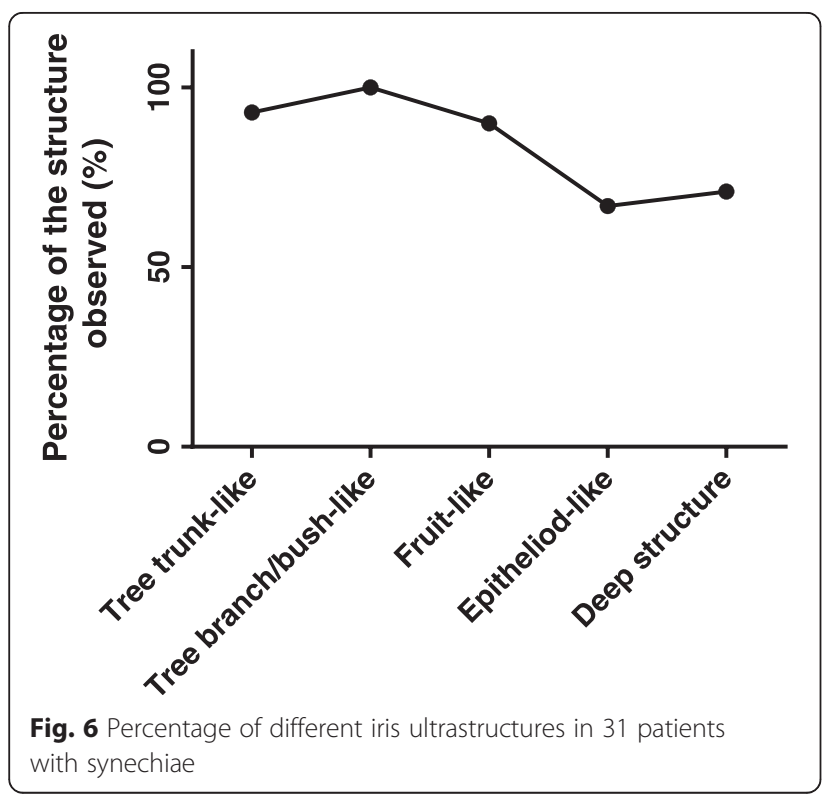

cases the pigment particles fall off from the surface, likely resembling disease conditions with pigment particles falling from iris known as Tyndall's Phenomenon and keratic precipitates.

Taken together, in this study, the ultrastructure of iris contacting with cornea has been visualized using in vivo LSCM. Especially, among the structures, the scaffold structure of the iris surface and pigment clumps can be clearly observed. Since what we have observed are the in vivo ultrastructures of the iris under the conditions of iris-cornea contact, we expect that some of the structures are disease associated, while some represent the normal iris structure. Indeed, the fall-off of pigment particles observed by our in vivo assay may shed light on the mechanism underlying the Tyndall's phenomenon in iris-associated diseases. Since we only have 31 cases in this study, it is still too early to correlate the specific changes in the in vivo ultrastructure to specific irisassociated diseases.

\section{Conclusions}

Using in vivo LSCM, the ultrastructure of iris in patients with synechiae has been visualized. Five iris ultrastructures can be clearly observed, with some of the structures maybe disease-associated. Our study provides a non-invasive approach to visualize the ultrastructure of the iris, which sets up a foundation to visualize certain iris-associated diseases in the future.

\section{Ethics}

The study protocol was approved by internal review board (IRB) of Shenzhen Eye Hospital.

\section{Consent to publish}

Informed consent was obtained from the patients for the study. Since the study contains no individual persons' data, there is no requirement for consent to publish. 


\section{Availability of data and materials}

All the data supporting your findings is contained within the manuscript.

\section{Additional file}

Additional file 1: Table S1. Detailed structures observed in individual patients with synechaie. (DOCX $13 \mathrm{~kb}$ )

\section{Abbreviations}

LSCM: Laser scanning confocal microscopy; UBM: Ultrasound biomicroscopy; OCT: Optical coherence tomography.

\section{Competing interests}

The authors declare that they have no competing interests.

\section{Authors' contributions}

M. L. contributions significantly to the conception, design, analysis and interpretation of data; H. C. and S. T. contributions significantly to the acquisition of data; P. G. contributions significantly to data analysis; C. Z contributions to data interpretation; S. W. contributes to the intellectual content of the project, interpretation of the data and manuscript writing. All the authors have read and approved of the final version of the manuscript.

\section{Acknowledgements}

The authors wish to thank Dr. Xiaoming Yao of Shenzhen Eye Hospital for helpful collection of cases.

\section{Funding}

Ming Li, Hongbo Cheng, Guo Ping and Chun Zhang were supported by Shenzhen Science and Technology Innovation Awards (JCYJ20130401152829824, CXZZ20130516161815191); Confocal microscopy instrumentation was provided by Heidelberg Engineering; Shusheng Wang was supported by Startup fund from Tulane University, NIH Grant EY021862 a career development award from the Research to Prevent Blindness foundation, and a Bright Focus Foundation Award in Age-related Macular Degeneration.

\section{Author details}

${ }^{1}$ Key laboratory of ophthalmology, Shenzhen Eye Hospital, Ji-nan University, Shenzhen 518000, P. R. China. ${ }^{2}$ Department of Cell and Molecular Biology, Tulane University, 2000 Percival Stern Hall, 6400 Freret Street, New Orleans, LA 70118, USA. ${ }^{3}$ Department of Ophthalmology, Tulane University, 1430 Tulane Avenue, SL-69, New Orleans, LA 70112, USA.

Received: 24 June 2015 Accepted: 15 April 2016

Published online: 26 April 2016

\section{References}

1. Gritz DC, Wong IG. Incidence and prevalence of uveitis in Northern California: the Northern California Epidemiology of Uveitis Study. Ophthalmology. 2004;111(3):491-500. PubMed: 15019324

2. Sbeity Z, Palmiero PM, Tello C, et al. Noncontact in vivo confocal laser scanning microscopy of exfoliation syndrome. Trans Am Ophthalmol Soc. 2008:106(3):46-55. PubMed: 19277220.

3. Tavakoli M, Hossain P, Malik RA. Clinical applications of corneal confocal microscopy. Clin Ophthalmol. 2008:2(2):435-45. PubMed: 19668734.

4. Kaufman SC, Beuerman RW, Kaufman HE. Diagnosis of advanced Fuchs' endothelial dystrophy with the confocal microscope. Am J Ophthalmol. 1993;116(5):652-3. PubMed: 8238235

5. Chiou AG, Kaufman SC, Beuerman RW, Ohta T, et al. Confocal microscopy in the iridocorneal endothelial syndrome. Br J Ophthalmol. 1999;83(6):697-702 PubMed: 10340979.

6. Chiou AG, Cadez R, Böhnke M. Diagnosis of Dieffenbachia induced corneal injury by confocal microscopy. Br J Ophthalmol. 1997;81(2):168-9. PubMed: 9059255.

7. Kaufman SC, Chew SJ, Capps SC, et al. Confocal microscopy of corneal penetration by tarantula hairs. Scanning. 1994;16(5):312-5. PubMed: 7994494.
8. Cavanagh HD, McCulley JP. In vivo confocal microscopy and acanthamoeba keratitis. Am J Ophthalmol. 1996:121(2):207-8. PubMed: 8623891

9. Chew SJ, Beuerman RW, Assouline M, et al. Early diagnosis of infectious keratitis with in vivo real time confocal microscopy. CLAO J. 1992:18(3):197-201. PubMed: 1499129

10. Florakis GJ, Moazami G, Schubert H, et al. Scanning slit confocal microscopy of fungal keratitis. Arch Ophthalmol. 1997;115(11):1461-3. PubMed: 9366681

11. Pfister DR, Cameron JD, Krachmer JH, et al. Confocal microscopy findings of acanthamoeba keratitis. Am J Ophthalmol. 1996;121(2):119-28. PubMed: 8623881.

12. Shah GK, Pfister D, Probst LE, et al. Diagnosis of microsporidial keratitis by confocal microscopy and the chromatrope stain. Am J Ophthalmol. 1996; 121(1):89-91. PubMed: 8554085.

13. Sutphin JE, Kantor AL, Mathers WD, et al. Evaluation of infectious crystalline keratitis with confocal microscopy in a case series. Cornea. 1997:16(1):21-6. PubMed: 8985629

14. Sbeity Z, Palmiero PM, Tello C, et al. Non-contact in vivo confocal scanning laser microscopy in exfoliation syndrome, exfoliation syndrome suspect and normal eyes. Acta Ophthalmol. 2011;89(3):241-7. PubMed:19860778.

15. Winchester K, Mathers WD, Sutphin JE. Diagnosis of Aspergillus keratitis in vivo with confocal microscopy. Cornea. 1997;16(1):27-31. PubMed: 8985630.

16. Lim LL, Hoang L, Wong T, et al. Intravital microscopy of leukocyte-endothelial dynamics using the Heidelberg confocal laser microscope in scleritis and allergic conjunctivitis. Mol Vis. 2006:12(10):1302-5. PubMed: 17110913.

17. Wang N, Lai M, Zhou W. Quantitative real time measurement of iris configuration in human eyes. Yan Ke Xue Bao. 1997:13(1):29-34. PubMed: 11189323

18. Garibaldi DC, Schein OD, Jun A. Features of the iridocorneal endothelial syndrome on confocal microscopy. Cornea. 2005;24(3):349-51. PubMed: 15778613.

19. Amos WB, White JG. How the Confocal Laser Scanning Microscope entered Biological Research. Biol Cell. 2003;95(6):335-42. PubMed: 14519550

20. Grupcheva CN, McGhee CN, Dean S, Craig JP. In vivo confocal microscopic characteristics of iridocorneal endothelial syndrome. Clin Experiment Ophthalmol. 2004:32(3):275-83. PubMed: 15180840.

21. Alvarado JA, Murphy CG, Juster RP, et al. Pathogenesis of Chandler's syndrome, essential iris atrophy and the Cogan-Reese syndrome. I. Alterations of the corneal endothelium. Invest Ophthalmol Vis Sci. 1986: 27(6):853-72. PubMed: 3710729.

22. Góes RM, Barbosa FL, de Faria-E-Sousa SJ, et al. Proliferation of the vascular endothelium of the iris following total debridement of the corneal epithelium and limbal excision of rabbits. Graefes Arch Clin Exp Ophthalmol. 2008;246(7): 999-1007. PubMed: 18431590

\section{Submit your next manuscript to BioMed Central} and we will help you at every step:

- We accept pre-submission inquiries

- Our selector tool helps you to find the most relevant journal

- We provide round the clock customer support

- Convenient online submission

- Thorough peer review

- Inclusion in PubMed and all major indexing services

- Maximum visibility for your research

Submit your manuscript at www.biomedcentral.com/submit 